PRE-PRINT CTP $\sharp 2629$

APRIL 1997

hep-th/9704206

\title{
Supersymmetric Yang-Mills Theory and Riemannian Geometry
}

\author{
RICARDO SCHIAPPA \\ Center for Theoretical Physics and Department of Physics \\ Massachusetts Institute of Technology, 77 Massachusetts Avenue \\ Cambridge, MA 02139, U.S.A. \\ E-mail: RICARDOs@MIT.EDU
}

\begin{abstract}
We introduce new local gauge invariant variables for $N=1$ supersymmetric Yang-Mills theory, explicitly parameterizing the physical Hilbert space of the theory. We show that these gauge invariant variables have a geometrical interpretation, and can be constructed such that the emergent geometry is that of $N=1$ supergravity: a Riemannian geometry with vector-spinor generated torsion. Full geometrization of supersymmetric Yang-Mills theory is carried out, and geometry independent divergences associated to the inversion of a differential operator with zero modes - that were encountered in the non-supersymmetric case - do not arise in this situation.
\end{abstract}

PACS: 02.40.Ky, 11.15.Tk, 11.30.Pb

Keywords: Supersymmetric Yang-Mills Theory, Gauge Invariance, Geometric Variables, Gauss' law 


\section{Introduction}

For quite sometime now, there exists a nice geometrical setting for Yang-Mills theory. That is based on fiber bundle differential geometry, where the configuration space is obtained by factoring out time independent gauge transformations, and is then seen as the base space of a principal fiber bundle, where the structure group is the gauge group [1]. There are many concepts of Riemannian geometry that can then came into the game, as there is the possibility of defining a Riemannian metric on the space of non-equivalent gauge connections [2].

However, this setting must be cast into a more workable form when we want to study the strong coupling regime of Yang-Mills theory. In here, gauge invariance becomes an important constraint on states of the theory in the form of Gauss' law. This constraint amounts to a reduction of the number of degrees of freedom present in the gauge connection: if we start with a gauge group $G$, in the canonical formalism and in temporal gauge $A_{0}^{a}=0$, the number of variables is $3 \operatorname{dim} G$, when in fact we only have $2 \operatorname{dim} G$ physical gauge invariant degrees of freedom. The question of whether one can construct local gauge invariant variables is then an important one, as it would allow us to easily implement the Gauss' law constraint. These variables would then seem the most appropriate ones to describe the physical space of the theory. Moreover, observe that in temporal gauge the remaining local gauge invariance is now restricted to space-dependent transformations at a fixed time. This is the true quantum mechanical symmetry of the theory. Working with local gauge invariant variables, this symmetry of the Hamiltonian can be maintained exactly, even under approximations to the dynamics.

This idea first appeared in [3] [4], and has recently gained new momentum with the work in [5] [6] [7] [8] [9] [10] [11] [12] [13], and references therein. In [8], one constructs a change of variables that will allow us to replace the coordinates $A_{i}^{a}$ by new coordinates $u_{i}^{a}$ that have the property of transforming covariantly under the gauge group, as opposed to as a gauge connection. Then, in these new coordinates, the generator of gauge transformations becomes a (color) rotation generator, and by contracting in color we can obtain gauge invariant variables to our theory, $g_{i j} \equiv u_{i}^{a} u_{j}^{a}$. States $\Psi\left[g_{i j}\right]$ depending only on these gauge invariant variables manifestly satisfy Gauss' law. We must be careful, however. Not any choice of gauge covariant variables is adequate: an appropriate set of variables should describe the correct number of gauge invariant degrees of freedom at each point of space, and should also be free of ambiguities such as $\mathrm{Wu}$-Yang ambiguities [14]. In this case, several gauge unrelated vector potentials may lead to the same color magnetic field. Variables that are $\mathrm{Wu}$-Yang insensitive are of no use, as in the functional integral formulation Wu-Yang related potentials must be integrated over - since they are not gauge related -, while functional integration over $\mathrm{Wu}$-Yang insensitive variables always misses these configurations. The absence of Wu-Yang ambiguities will be clear if we are able to invert the variable transformation, i.e., if when transforming $A \rightarrow u$ we can also have an explicit expression for $A[u]$. 
In [8], the set of gauge covariant variables $\left\{u_{i}^{a}\right\}$ that replace the $S U(2)$ gauge connection was defined by the differential equations:

$$
\epsilon^{i j k} D_{j} u_{k}^{a} \equiv \epsilon^{i j k}\left(\partial_{j} u_{k}^{a}+\epsilon^{a b c} A_{j}^{b} u_{k}^{c}\right)=0
$$

which is equivalent to writing,

$$
\partial_{j} u_{k}^{a}+\epsilon^{a b c} A_{j}^{b} u_{k}^{c}-\Gamma_{j k}^{s} u_{s}^{a}=0
$$

as the $\left\{u_{i}^{a}\right\}$ have det $u \neq 0$, and so form a complete basis. Observe that we have $\Gamma_{j k}^{s}=\Gamma_{k j}^{s}$, and these quantities can be written as,

$$
\Gamma_{j k}^{s}=\frac{1}{2} g^{s n}\left(\partial_{j} g_{n k}+\partial_{k} g_{j n}-\partial_{n} g_{j k}\right)
$$

where

$$
g_{i j} \equiv u_{i}^{a} u_{j}^{a}
$$

So, a "metric" tensor was implicitly introduced by the defining equations for the new variables, (1.1). Observe that equation (1.2) is simply the so-called dreibein postulate, where the $\left\{u_{i}^{a}\right\}$ plays the role of a dreibein, $\omega_{i}^{a c} \equiv \epsilon^{a b c} A_{i}^{b}$ is a spin-connection, and $\Gamma_{j k}^{i}$ is the affine metric connection. A torsion-free Riemannian geometry in a three manifold was then introduced by the definition of the new variables. The metric $g_{i j}$ contains in itself the six local gauge invariant degrees of freedom of the $S U(2)$ gauge theory. Moreover, any gauge invariant wave-functional of $A_{i}^{a}$ can be written as a function of $g_{i j}$ only, and any wave-functional of $g_{i j}$ is gauge invariant [8]. This implements gauge invariance exactly. Finally, the dreibein postulate can be inverted so that we obtain,

$$
A_{i}^{a}=-\frac{1}{2} \epsilon^{a b c} u^{b j} \nabla_{i} u_{j}^{c}
$$

where we use the notation $\nabla_{j} u_{k}^{a} \equiv \partial_{j} u_{k}^{a}-\Gamma_{j k}^{s} u_{s}^{a}$ for the purely geometric covariant derivative (as opposed to the gauge covariant derivative). Therefore, the new variables avoid Wu-Yang ambiguities.

Full geometrization of Yang-Mills theory in this formulation was then carried out in [8] [10]. The electric energy involves the inversion of a differential operator that can generically have zero modes. By deforming equations (1.1) it was then shown how one could proceed to compute the electric tensor [12]. Instanton and monopole configurations have been identified as the $S^{3}$ and $S^{2} \times \mathbf{R}$ geometries [12], and, more recently, the form the wave-functional for two heavy color sources should take has been calculated [13]. The computations are carried out in the Schroedinger representation of gauge theory, see [15] for a review.

Supersymmetric Yang-Mills theory has also been well established for quite sometime now. It allows for many simplifications in quantum computations, and with an appropriate choice of matter content and/or number of supersymmetry generators, we can obtain finite quantum field theories. Textbook references are [16] [17] [18]. 
Moreover, recently there has been a lot of progress and activity in the field due to the possibility of actually solving for the low-energy effective action of certain cases of supersymmetric Yang-Mills theories, starting with the work in [19]. It is then natural to extend the work on gauge invariant geometrical variables to the supersymmetric case. That is what we will do here.

We will see that it is possible to define variables that also have a geometrical interpretation, namely, as the variables present in supergravity. We should point out, however, that no coupling to gravity is ever considered. Still, we need a motivation to construct the new variables. As in the pure Yang-Mills case the new variables and geometry have an interpretation as the variables and geometry of three dimensional gravity, it is natural to assume that in the supersymmetric case the new variables and geometry could likewise have an interpretation as the variables and geometry of three dimensional supergravity. This will be a guiding principle throughout our work. More geometrical intuition on how to construct the new variables will come from an extra symmetry enjoyed by both the canonical variables and Gauss' law generator. That is a symmetry under $G L(3)$ transformations, a diffeomorphism symmetry. This will allow us to naturally assign tensorial properties to diverse local quantities of the theory. Obviously the Hamiltonian (or any other global operator) will not possess this symmetry. After all, supersymmetric Yang-Mills theory is not diffeomorphism invariant.

The plan of this paper is as follows. In section 2 we start by reviewing the conventions of $N=1$ supersymmetry, and also outline the geometry of supergravity. In section 3 we will then explore the $G L(3)$ symmetry, assigning tensorial properties to local (composite) operators. We this in hand, we then proceed to define gauge invariant geometrical variables for supersymmetric Yang-Mills theory in section 4, carrying out the full geometrization of the theory in section 5. Section 6 presents a concluding outline.

\section{Review and Conventions}

The conventions in [16] [17] and [18] are basically the same. We will follow [16] with minor changes, as we take $\sigma^{\mu \nu}=\frac{1}{4}\left[\gamma^{\mu}, \gamma^{\nu}\right]$. The $N=1$ supersymmetry algebra is obtained by introducing one spinor generator, $Q$, which is a Majorana spinor, to supplement the usual (bosonic) generators of the Poincaré group. The $N=1$ supersymmetry algebra is then the Poincaré algebra plus:

$$
\begin{gathered}
{\left[P_{\mu}, Q\right]=0} \\
{\left[M_{\mu \nu}, Q\right]=-i \sigma_{\mu \nu} Q} \\
\{Q, \bar{Q}\}=2 \gamma^{\mu} P_{\mu}
\end{gathered}
$$

where $\bar{Q} \equiv Q \gamma^{0}$. 
Supersymmetric gauge theory, based on gauge group $G$ with gauge algebra $\mathcal{G}$, has as component fields the gluons, or gauge connection, $A_{\mu}^{a}$; the gluinos, super-partners of the gauge fields and Majorana spinors, $\lambda^{a}$; and the scalar auxiliary fields $D^{a}$. All these fields are in the adjoint representation of $\mathcal{G}$. In Wess-Zumino gauge, the action is,

$$
S=\int d^{4} x\left\{-\frac{1}{4} F_{\mu \nu}^{a} F^{a \mu \nu}+\frac{i}{2} \bar{\lambda}^{a} \gamma^{\mu} D_{\mu} \lambda^{a}+\frac{1}{2} D^{a} D^{a}\right\}
$$

where we can see that the auxiliary fields have no dynamics. The supersymmetry transformation laws of the fields, that leave the action invariant are:

$$
\begin{gathered}
\delta A_{\mu}^{a}=i \bar{\varepsilon} \gamma_{\mu} \lambda^{a} \\
\delta \lambda^{a}=\left(\sigma^{\mu \nu} F_{\mu \nu}^{a}-i \gamma_{5} D^{a}\right) \varepsilon \\
\delta D^{a}=\bar{\varepsilon} \gamma_{5} \gamma^{\mu} D_{\mu} \lambda^{a}
\end{gathered}
$$

where $\varepsilon$ is a Majorana spinor, which is the parameter of the infinitesimal supersymmetry transformation. These transformation laws implement a representation of the $N=1$ supersymmetry algebra in the quantum gauge field theory. The Noether conserved current of supersymmetry is a vector-spinor,

$$
J^{\mu}=i \gamma^{\mu} \sigma^{\alpha \beta} F_{\alpha \beta}^{a} \lambda^{a}
$$

and so the quantum field theoretic representation of the supersymmetry generator is given by the Majorana spinor,

$$
Q=i \int d^{3} x \gamma^{0} \sigma^{\mu \nu} F_{\mu \nu}^{a} \lambda^{a}
$$

This outlines our usage of notation for supersymmetric gauge theory. We still have to outline notation for the supergravity geometry. In here, we will have a graviton, $g_{\mu \nu}$, and a gravitino, which is described by a Rarita-Schwinger field, $\psi_{\mu}$. So, we need to start by reviewing notation for inserting spinors in curved manifolds. Having a metric, we can define orthonormal frames, and so insert a tetrad base at the tangent space to a given point, which will allow us to translate between curved and flat indices. In particular, this allows us to introduce gamma matrices in the manifold, and so introduce spinors. If we are in a manifold $M$, and pick a point $p \in M$, we can introduce a tetrad base $\left\{u_{\mu}^{a}\right\}$ at $p$ via,

$$
g_{\mu \nu}=u_{\mu}^{a} u_{\nu}^{b} \eta_{a b}
$$

defining an orthonormal frame at each point on $M$. We can now insert gamma matrices as $\gamma^{\mu}(x) u_{\mu}^{a}(x)=\gamma^{a}$, where the $\gamma^{a}$ are numerical matrices. Local Lorentz transformations in the tangent space $T_{p} M$ are $\Lambda_{b}^{a}(p)$ and (Dirac) spinors at $p \in M$ rotate as,

$$
\psi_{\alpha}(p) \rightarrow S_{\alpha \beta}\left(\Lambda_{b}^{a}(p)\right) \psi_{\beta}(p)
$$


Now, we construct a covariant derivative, $\mathbf{D}_{a} \psi_{\alpha}$, which is a local Lorentz vector, and transforms as a spinor,

$$
\mathbf{D}_{a} \psi_{\alpha} \rightarrow S_{\alpha \beta}(\Lambda) \Lambda_{a}^{b} \mathbf{D}_{b} \psi_{\beta}
$$

That is done via a connection $\Omega_{\mu}$ such that, $\mathbf{D}_{a} \psi=u_{a}^{\mu}\left(\partial_{\mu}+\Omega_{\mu}\right) \psi$, and,

$$
\Omega_{\mu}=\frac{1}{2} \omega_{\mu}^{a b} \sigma_{a b}=\frac{1}{2} u_{\nu}^{a} \nabla_{\mu} u^{b \nu} \sigma_{a b}
$$

where $\omega_{\mu}^{a b}$ is the spin-connection.

Now that we have spinors defined on curved manifolds, we can proceed with supergravity. In here, the Riemannian connection $\Gamma_{\mu \nu}^{\rho}$ is not torsion-free. It is still metric compatible, so that we can write,

$$
\Gamma_{\mu \nu}^{\rho}=\hat{\Gamma}_{\mu \nu}^{\rho}-K_{\mu \nu}^{\rho}
$$

where $\hat{\Gamma}_{\mu \nu}^{\rho}$ is the affine metric connection, and $K_{\mu \nu}{ }^{\rho}$ is the contorsion tensor. Hatted symbols will always stand for quantities computed via the affine metric connection. The torsion tensor is,

$$
T_{\mu \nu}^{\rho} \equiv \Gamma_{\mu \nu}^{\rho}-\Gamma_{\nu \mu}^{\rho}
$$

and so,

$$
K_{\mu \nu}{ }^{\rho}=-\frac{1}{2}\left(T_{\mu \nu}{ }^{\rho}-g_{\nu \lambda} g^{\sigma \rho} T_{\mu \sigma}^{\lambda}-g_{\mu \lambda} g^{\sigma \rho} T_{\nu \sigma}{ }^{\lambda}\right)
$$

In $N=1$ supergravity, the torsion is defined by the Rarita-Schwinger field $\psi_{\mu}$ as,

$$
T_{\mu \nu}^{a}=\frac{i}{2} k^{2} \bar{\psi}_{\mu} \gamma^{a} \psi_{\nu}
$$

where $a$ is a flat index, and $k$ is the gravitational dimensionfull constant. The tetrad postulate is,

$$
\mathcal{D}_{\mu} u_{\nu}^{a} \equiv \partial_{\mu} u_{\nu}^{a}+\omega_{\mu}^{a b} u_{b \nu}-\Gamma_{\mu \nu}^{\rho} u_{\rho}^{a}=0
$$

and the covariant derivative acting on spinor indices is,

$$
\left(\mathbf{D}_{\mu}\right)_{\alpha \beta} \equiv \delta_{\alpha \beta} \partial_{\mu}+\frac{1}{2} \omega_{\mu a b}\left(\sigma^{a b}\right)_{\alpha \beta}
$$

Finally, the supersymmetry transformations that leave the $N=1$ supergravity action (Einstein-Hilbert plus Rarita-Schwinger) invariant are:

$$
\begin{gathered}
\delta u_{\mu}^{a}(x)=i k \bar{\xi}(x) \gamma^{a} \psi_{\mu}(x) \\
\delta \psi_{\mu}(x)=\frac{2}{k} \mathbf{D}_{\mu} \xi(x)
\end{gathered}
$$

where $\xi(x)$ is the infinitesimal parameter of the transformation (now a space-time dependent Majorana spinor), and where we have not included the auxiliary fields. This ends our review and outline of conventions. We can now start analyzing the gauge invariant variables geometrization of supersymmetric Yang-Mills theory. 


\section{Canonical Formulation and $G L(3)$ Properties}

In the Lagrangian formulation of the theory, the $N=1$ supersymmetry algebra closes only up to the field equations. In order to obtain manifest supersymmetry, and offshell closure of the algebra, we need to introduce auxiliary fields. In contrast to this situation, it is known that in the canonical formalism the $N=1$ super Lie algebra closes without the introduction of auxiliary fields (in terms of Dirac brackets the algebra closes strongly; otherwise it closes weakly, i.e., up to the first-class constraints) 20] [21]. So, we drop the auxiliary fields.

The Hamiltonian for supersymmetric gauge theory is therefore,

$$
H=\int d^{3} x\left\{\frac{1}{2} e^{2}\left(E^{a i}\right)^{2}+\frac{1}{2 e^{2}}\left(B^{a i}\left[A_{j}^{b}\right]\right)^{2}-\frac{i}{2} \bar{\lambda}^{a} \gamma^{i} D_{i} \lambda^{a}\right\}
$$

where $e$ is the coupling constant. The gauge covariant derivative is,

$$
D_{i} \lambda^{a}=\partial_{i} \lambda^{a}+f^{a b c} A_{i}^{b} \lambda^{c}
$$

and the magnetic field potential energy,

$$
B^{a i}\left[A_{n}^{b}\right] \equiv \frac{1}{2} \epsilon^{i j k} F_{j k}^{a}\left[A_{n}^{b}\right]=\epsilon^{i j k}\left(\partial_{j} A_{k}^{a}+\frac{1}{2} f^{a b c} A_{j}^{b} A_{k}^{c}\right)
$$

We still have to impose the Gauss' law constraint on the physical states of the theory,

$$
\mathcal{G}^{a}(x) \equiv D_{i} E^{a i}(x)-\frac{i}{2} f^{a b c} \lambda^{b}(x) \lambda^{c}(x) \quad, \quad \mathcal{G}^{a}(x) \Psi\left[A_{i}^{b}, \lambda^{c}\right]=0
$$

This local composite operator is the generator of local gauge transformations.

There is one more element in the $N=1$ supersymmetric Yang-Mills theory, and that is the Majorana spinor $Q$, the generator of supersymmetry. Using the definition,

$$
Q \equiv \int d^{3} x \mathcal{Q}(x)
$$

we can then write,

$$
\mathcal{Q}(x)=i\left(-e \gamma_{i} E^{a i}(x)+\frac{1}{e} \epsilon_{i j k} \gamma^{0} \sigma^{i j} B^{a k}\left[A_{n}^{b}(x)\right]\right) \lambda^{a}(x)
$$

or, using the explicit Weyl representation of the gamma matrices, we can equivalently write this local composite operator in a more compact form,

$$
\mathcal{Q}(x)=i\left(\begin{array}{cc}
0 & \left(e E^{a i}(x)+\frac{i}{e} B^{a i}\left[A_{n}^{b}(x)\right]\right) \sigma_{i} \\
0 & \left.0 E^{a i}(x)+\frac{i}{e} B^{a i}\left[A_{n}^{b}(x)\right]\right) \sigma_{i}
\end{array}\right.
$$

Now, in the bosonic half of the theory, the canonical variables are $A_{i}^{a}(x)$ and $E^{a i}(x)$. Canonical quantization is carried out by the commutator,

$$
\left[A_{i}^{a}(x), E^{b j}(y)\right]=i \delta^{a b} \delta_{j}^{i} \delta(x-y)
$$


The momentum $E^{a i}(x)$ will be implemented as a functional derivative acting on wavefunctionals as,

$$
E^{a i}(x) \Psi\left[A_{n}^{b}, \lambda^{c}\right] \rightarrow-i \frac{\delta}{\delta A_{i}^{a}(x)} \Psi\left[A_{n}^{b}, \lambda^{c}\right]
$$

In the fermionic half of the theory, we have a Majorana spinor $\lambda^{a}(x)$. Canonical quantization is carried out by establishing the anti-commutation relations for the spinorial field,

$$
\left\{\lambda_{\alpha}^{a}(x), \lambda_{\beta}^{b}(y)\right\}=\delta^{a b} \delta_{\alpha \beta} \delta(x-y)
$$

Both the commutator and the anti-commutator are to be evaluated at equal times. We can now compute the commutators and anti-commutators of this theory, which involve the composite operators $H, \mathcal{G}^{a}(x)$ and $\mathcal{Q}(x)$. Clearly, these (anti)-commutators are related to the symmetry transformations generated by these operators.

The commutators involving the generator of local gauge transformations of the canonical variables can be computed to be,

$$
\begin{gathered}
{\left[A_{i}^{a}(x), \mathcal{G}^{b}(y)\right]=i\left(\delta^{a b} \partial_{i}-f^{a c b} A_{i}^{c}(x)\right) \delta(x-y)} \\
{\left[E^{a i}(x), \mathcal{G}^{b}(y)\right]=i f^{a b c} E^{c i}(x) \delta(x-y)} \\
{\left[\lambda^{a}(x), \mathcal{G}^{b}(y)\right]=i f^{a b c} \lambda^{c}(x) \delta(x-y)}
\end{gathered}
$$

and the (anti)-commutators involving the local composite operator associated to the supersymmetry generator can similarly be found to be,

$$
\begin{gathered}
{\left[A_{i}^{a}(x), \mathcal{Q}(y)\right]=-\gamma_{i} \lambda^{a}(x) \delta(x-y)} \\
{\left[E^{a i}(x), \mathcal{Q}(y)\right]=\epsilon^{i j k}\left(\epsilon_{k n m} \gamma^{0} \sigma^{n m}\right) D_{j} \lambda^{a} \delta(x-y)} \\
\left\{\lambda^{a}(x), \mathcal{Q}(y)\right\}=i\left(-e \gamma_{i} E^{a i}(x)+\frac{1}{e} \epsilon_{i j k} \gamma^{0} \sigma^{i j} B^{a k}\left[A_{n}^{b}(x)\right]\right) \delta(x-y)
\end{gathered}
$$

Moreover, we will also have that the Hamiltonian and the supersymmetry generator are both gauge invariant composite operators, as,

$$
\begin{gathered}
{\left[H, \mathcal{G}^{a}(x)\right]=0} \\
{\left[\mathcal{Q}(x), \mathcal{G}^{a}(y)\right]=0}
\end{gathered}
$$

As expected, the generators $\mathcal{G}^{a}(x)$ define the local gauge algebra,

$$
\left[\mathcal{G}^{a}(x), \mathcal{G}^{b}(y)\right]=i f^{a b c} \mathcal{G}^{c}(x) \delta(x-y)
$$

The supersymmetry generator $Q$ defines, along with the generators of the Poincaré algebra, the $N=1$ supersymmetry algebra [20]. However, the defined local composite operator $\mathcal{Q}(x)$ does not define a local algebra. That is to be expected as we do not have local supersymmetry in our theory. This local operator was only introduced in order 
to facilitate the following tensorial analysis based on diffeomorphism transformations of the presented (anti)-commutators.

So, we now want to check that there is a $G L(3)$ symmetry at work for the formulae (3.8), (3.10) and (3.11-13), (3.19). The bosonic part tensorial assignments will be just like in the pure Yang-Mills case [\&], as is to be expected. So, the mentioned canonical relations are covariant under diffeomorphisms $x^{i} \rightarrow y^{n}\left(x^{i}\right)$ on the domain $\mathbf{R}^{3}$, provided $A_{i}^{a}(x)$ is a one-form in $\mathbf{R}^{3}$, transforming as

$$
A_{n}^{\prime a}\left(y^{m}\right)=\frac{\partial x^{i}}{\partial y^{n}} A_{i}^{a}\left(x^{j}\right)
$$

where $\left[\partial x^{i} / \partial y^{n}\right]$ is a $G L(3)$ matrix. That $\mathbf{A}(x) \equiv A_{\mu}^{a}(x) T^{a} d x^{\mu}$ is a Lie algebra valued one-form is a well known fact from the fiber bundle geometry of gauge theory; so consistency holds. Also, provided $E^{a i}(x)$ is a vector density (weight -1 ) in $\mathbf{R}^{3}$, transforming as

$$
E^{\prime a n}\left(y^{m}\right)=\operatorname{det}\left[\frac{\partial x^{i}}{\partial y^{n}}\right] \frac{\partial y^{n}}{\partial x^{i}} E^{a i}\left(x^{j}\right)
$$

The same property holds for $B^{a k}(x)$. This is consistent with the implementation of $E^{a i}(x)$ as a functional derivative (3.9), and with the definition of the magnetic field (3.3). Commutator (3.8) is then clearly diffeomorphic invariant, without the intervention of a space metric. However, to introduce spinors, we do need a metric (more precisely, a dreibein base). We will assume we do have a metric, $g_{i j}$, and later we will construct it using the bosonic dynamical variables of the theory.

When restricted to three dimensional Euclidean space, Lorentz transformations become rotations in $\mathbf{R}^{3}$. The spinor representation of a rotation is then, at a point $p \in M$, given by the orthogonal matrix acting on spinor indices,

$$
S_{\alpha \beta}(\Lambda(p))=\exp \left(\frac{1}{2} \omega_{a b}(\Lambda(p)) \sigma^{a b}\right)_{\alpha \beta}
$$

where $\omega$ is the rotation parameter. We can now define the $G L(3)$ properties of $\lambda^{a}(x)$, in order to maintain the anti-commutator (3.10) diffeomorphism invariant. That relation is invariant under diffeomorphisms, provided $\lambda^{a}(x)$ is a spinorial density (weight $-\frac{1}{2}$ ) in $\mathbf{R}^{3}$, transforming as

$$
\lambda_{\alpha}^{\prime a}\left(y^{m}\right)=\operatorname{det}\left[\frac{\partial x^{i}}{\partial y^{n}}\right]^{\frac{1}{2}} S_{\alpha \beta}(\Lambda(p)) \lambda_{\beta}^{a}\left(x^{j}\right)
$$

Let us now see what are the consequences of these $G L(3)$ properties on the composite local operators $\mathcal{G}^{a}(x)$ and $\mathcal{Q}(x)$. Starting with the generator of local gauge transformations, we can observe that the tensorial properties of the canonical variables imply that under diffeomorphisms we will have that $\mathcal{G}^{a}(x)$ is a scalar density (weight -1 ) in $\mathbf{R}^{3}$, transforming as

$$
\mathcal{G}^{\prime a}\left(y^{m}\right)=\operatorname{det}\left[\frac{\partial x^{i}}{\partial y^{n}}\right] \mathcal{G}^{a}\left(x^{j}\right)
$$


This automatically verifies that the canonical commutators (3.11-13) and (3.19) are invariant under local diffeomorphisms on the domain of the local canonical variables.

Now, look at the other local composite operator, $\mathcal{Q}(x),(3.6)$ or (3.7). First observe that in (3.7) the Pauli matrices $\vec{\sigma}$ are numerical matrices, and not dynamical ones (in which case we would write $\sigma^{i}(x) u_{i}^{a}(x)=\sigma^{a},\left\{u_{i}^{a}\right\}$ being a dreibein base). We write (3.7) as:

$$
\mathcal{Q}(x) \equiv \sigma_{i} \otimes \amalg^{i}(x)
$$

where,

$$
\amalg^{i}(x) \equiv i\left(\begin{array}{cc}
0 & e E^{a i}(x)+\frac{i}{e} B^{a i}\left[A_{n}^{b}(x)\right] \\
-e E^{a i}(x)+\frac{i}{e} B^{a i}\left[A_{n}^{b}(x)\right] & 0
\end{array}\right) \lambda^{a}(x)
$$

Then, the tensorial properties under $G L(3)$ of the canonical variables imply that, under diffeomorphisms, we will have that $\amalg^{i}(x)$ is a vector-spinor density (weight $-\frac{3}{2}$ ) in $\mathbf{R}^{3}$, transforming as

$$
\amalg_{\alpha}^{\prime n}\left(y^{m}\right)=\operatorname{det}\left[\frac{\partial x^{i}}{\partial y^{n}}\right]^{\frac{3}{2}} \frac{\partial y^{n}}{\partial x^{i}} S_{\alpha \beta}(\Lambda(p)) \amalg_{\beta}^{i}\left(x^{j}\right)
$$

However, as the $\sigma_{i}$ 's in (3.25) are numerical, they do not transform under the diffeomorphism, and so $\mathcal{Q}(x)$ fails to be covariant. This is to be expected, as we will see below.

So, the $G L(3)$ symmetry of the (anti)-commutation relations involving local (composite) operators and local variables has been established, given the tensorial properties assigned to the canonical variables. Clearly, the theory itself fails to be $G L(3)$ invariant, and that is to be expected: the Hamiltonian is not covariant under diffeomorphisms (the metric $\delta_{i j}$ appears instead of $g_{i j}$, the measure $d^{3} x$ appears instead of $\sqrt{g} d^{3} x$, etc.). This can be related to the lack of covariance of the supersymmetry generator (3.25), (3.27). Indeed, we can see $\mathcal{Q}(x)$ as the square root of the Hamiltonian; so if the Hamiltonian fails to be covariant, so should the supersymmetry generator. Moreover, observe that when we square (3.25) we will obtain a term like $\sigma_{i} \sigma_{j}=\delta_{i j}+i \epsilon_{i j k} \sigma_{k}$, and this can be seen as the origin of the "wrong" metric $\delta_{i j}$ in the Hamiltonian, which will destroy the possibility of local covariance. Also, no global (composite) operator can have this $G L(3)$ symmetry, due to the "wrong" choice of integration measure. Now that we have assigned tensorial properties to local quantities in our supersymmetric theory, we are ready to proceed in looking for further geometrization in this canonical framework.

\section{Geometric Variables}

We will now limit ourselves to the simplest case of non-abelian gauge group, namely, $G=S U(2)$. Then, the structure constants are simply $f^{a b c}=\epsilon^{a b c}$. We will assume knowledge of the previous work done for pure Yang-Mills theory [8] [10] 12. 
We will want to have a representation of supersymmetry once we are in the new variables. As we know from the bosonic case [8], the gluon field is transformed into a "metric" field. The supersymmetry representation that includes a metric field is that of supergravity, and it also includes a vector-spinor field. So, we will expect that the gluino field will be transformed into a "gravitino" field. We will therefore want to transform the supersymmetric Yang-Mills variables, $\left\{A_{i}^{a}(x), \lambda^{a}(x)\right\}$, into the variables of three dimensional supergravity, $\left\{g_{i j}(x), \psi_{k}(x)\right\}$. We will also expect to obtain a geometry similar to the one of supergravity. After all, the defining equation for the $\left\{u_{i}^{a}(x)\right\}$ variables identifies them with a dreibein base in a three dimensional manifold.

Recall form section 2 what we are to expect. The geometry will have torsion, defined as,

$$
T_{i j}^{a}=\frac{i}{2} \bar{\psi}_{i} \gamma^{a} \psi_{j}
$$

We can insert a dreibein base through,

$$
g_{i j}=u_{i}^{a} u_{j}^{b} \delta^{a b}
$$

and we also expect that there could be some local supersymmetry transformation in these new variables, which we will call the "geometrical supersymmetry variation", and which would look like the supersymmetric transformation laws of supergravity,

$$
\begin{gathered}
\delta u_{i}^{a}(x)=i \bar{\xi}(x) \gamma^{a} \psi_{i}(x) \\
\delta \psi_{k}(x)=2 \mathbf{D}_{k} \xi(x)
\end{gathered}
$$

where the covariant derivative acting on spinor indices was defined in (2.15). With this at hand, the dreibein postulate is now written as,

$$
\mathcal{D}_{j} u_{k}^{a} \equiv \partial_{j} u_{k}^{a}+\omega_{j}^{a b} u_{k}^{b}-\Gamma_{j k}^{n} u_{n}^{a}=0
$$

also defining the symbol $\mathcal{D}_{j}$. Multiplying this equation by $\epsilon^{i j k}$, and defining the spin-connection via the gauge connection, as in,

$$
\omega_{j}^{a b}(x) \equiv \epsilon^{a c b} A_{j}^{c}(x)
$$

the dreibein postulate becomes,

$$
\epsilon^{i j k} D_{j} u_{k}^{a}=\epsilon^{i j k}\left(\partial_{j} u_{k}^{a}+\epsilon^{a b c} A_{j}^{b} u_{k}^{c}\right)=\frac{1}{2} \epsilon^{i j k} T_{j k}^{a}=\frac{i}{4} \epsilon^{i j k} \bar{\psi}_{j} \gamma^{a} \psi_{k}
$$

We will take these differential equations to define the change of variables $A_{i}^{a}(x) \rightarrow$ $u_{i}^{a}(x)$. Then, the reverse line of argument holds: the new variables $\left\{u_{i}^{a}(x)\right\}$ play the role of a dreibein, and from them we can construct a metric $g_{i j}=u_{i}^{a} u_{j}^{a}$ which is a local gauge invariant variable. The geometry defined by this new variable has torsion, 
given by (4.1). Clearly, for the change of variables to be well defined, we still need to specify what $\psi_{j}(x)$ is. That is the problem we will now address.

We begin with some dimensional analysis. We know that the gauge field $A_{i}^{a}(x)$ has mass dimension one, and the gaugino field $\lambda^{a}(x)$ has mass dimension three halfs. We also know that the mass dimension of the fermionic generator of the supersymmetry algebra is one half. Through definition (3.5) and expression (3.25) we observe that if we ever were to modify the gauge theory in order to covariantize it (inserting $\sigma^{i}(x) u_{i}^{a}(x)=\sigma^{a}$ in (3.25) and from then on), we would need the dreibein field to have zero mass dimension, as well as the metric. Though we are not going to modify the gauge theory in this work, we may as well stick to this broader perspective. Then, through the dreibein defining equation (4.6), we conclude that the "gravitino" field has mass dimension one half. These dimensional assignments are just like what happens in supergravity.

We can now see that this will have some influence on the construction of the "gravitino" defining equation. In fact, there are some a priori requirements for such an equation. It must be geometrical, either in a differential or algebraic way; we need 12 equations, to change the 12 variables $\lambda_{\alpha}^{a}$ to the 12 variables $\psi_{k \alpha}$; and the gluino field must be present in such an equation. If we moreover require linearity on fermionic variables (like we had linearity on the bosonic variables in (4.6)), we see that, by simple dimensional analysis, we can not write such an equation algebraically, but only differentially. Moreover, the equation is constrained to be of the form,

$$
\epsilon^{i j k} \mathbf{D}_{j} \psi_{k \alpha}=\mathcal{M}_{\alpha \beta}^{i a} \lambda_{\beta}^{a}
$$

where the matrix $\mathcal{M}_{\alpha \beta}^{i a}$ must have zero mass dimension, being so far otherwise arbitrary. However, we must be careful. Not only do we want to have a geometrical way in which to define the vector-spinor field, but we also want to be compatible with the fact that we are studying a supersymmetric theory. In particular, we would like the geometrical supersymmetry variation (4.3) to generate the gauge supersymmetry variation (2.3). So we will ask for the geometrical variation (4.3) to generate the gauge supersymmetry variation on the bosonic variables $A_{i}^{a}(x)$, and in the simplest case where $\xi(x) \equiv \varepsilon$.

Under a generic variation of the fields, we obtain for (4.6),

$$
\epsilon^{i j k} D_{j} \delta u_{k}^{a}=-\epsilon^{i j k} \epsilon^{a b c} u_{k}^{c} \delta A_{j}^{b}+\frac{1}{2} \epsilon^{i j k} \delta T_{j k}^{a}
$$

where,

$$
\frac{1}{2} \epsilon^{i j k} \delta T_{j k}{ }^{a}=\frac{i}{2} \epsilon^{i j k} \bar{\psi}_{j} \gamma^{a} \delta \psi_{k}
$$

The supersymmetry transformation laws we will need are (2.3) and (4.3). So, a supersymmetry transformation of the dreibein defining equation yields the "gravitino" defining equation. Performing the computations, based on the previous formulae, we 
are led to,

$$
\epsilon^{i j k} \mathbf{D}_{j} \psi_{k}=\epsilon^{i j k}\left(\partial_{j} \psi_{k}+\frac{1}{2} \omega_{j}^{a b} \sigma^{a b} \psi_{k}\right)=\frac{1}{3} \epsilon^{i j k} \epsilon^{a b c} \gamma^{a} \gamma_{j} \lambda^{b} u_{k}^{c}
$$

where $\gamma_{i}(x)=u_{i}^{a}(x) \gamma^{a}$.

We will take these differential equations to define the change of variables $\lambda^{a}(x) \rightarrow$ $\psi_{k}(x)$. Observe that this equation is precisely of the required form (4.7), and the matrix $\mathcal{M}_{\alpha \beta}^{i a}$ has been uniquely defined. Also, this alone guarantees that the geometric variation (4.3) will generate the bosonic gauge supersymmetry variation (2.3), when $\xi \equiv \varepsilon$. This does not guarantee however that the geometric variation will generate the fermionic gauge supersymmetry variation under the same circumstances. In fact, we can choose $\xi \equiv \xi[\varepsilon]$ through a differential equation (4.20) for $\xi$, such that the geometric variation generates the gauge supersymmetry variation on $\lambda^{a}(x)$, but we will not have $\xi \equiv \varepsilon$ in this case. This shows that even though we can generate the gauge supersymmetry variation via the geometrical supersymmetry variation under special circumstances, the geometric variation is not the original supersymmetry of Yang-Mills theory. The actual expressions for the supersymmetry variations on the new geometrical variables can nevertheless be computed using the usual expression,

$$
\delta \Phi=i[Q, \Phi]
$$

where $\Phi$ is any of the geometrical variables, and where we should express the supersymmetry generator in this geometric framework (see section 5). The resulting expressions would not be as simple as (2.3) or (4.3).

All together, we see that we can now define local gauge invariant geometric variables for supersymmetric Yang-Mills theory via the system of coupled non-linear partial differential equations, (4.6) and (4.10). These equations define a variable change $\left\{A_{i}^{a}, \lambda^{b}\right\} \rightarrow\left\{u_{i}^{a}, \psi_{k}\right\}$. They also introduce a three dimensional Riemannian geometry with torsion as given by (4.1-2) and (4.4).

Now that the definition of the new geometrical gauge invariant variables is concluded, we would like to invert the defining equations, in order to express $A_{i}^{a}(x)$ and $\lambda^{a}(x)$ in terms of the geometric variables. This inversion will make clear that there are no $\mathrm{Wu}$-Yang ambiguities related to these new variables. The defining equation for the dreibein (4.6) is equivalent to the dreibein postulate (4.4), where the connection is with torsion,

$$
\Gamma_{j k}^{n}=\hat{\Gamma}_{j k}^{n}-K_{j k}^{n}
$$

hatted symbols always denoting affine metric connection quantities. The contorsion tensor is computed from the torsion tensor, through (2.12), and we obtain,

$$
K_{i j n}=\frac{i}{4}\left(\bar{\psi}_{i} \gamma_{j} \psi_{n}+\bar{\psi}_{j} \gamma_{i} \psi_{n}-\bar{\psi}_{i} \gamma_{n} \psi_{j}\right)
$$

Define a purely geometric derivative through,

$$
\nabla_{j} u_{k}^{a} \equiv \partial_{j} u_{k}^{a}-\Gamma_{j k}^{n} u_{n}^{a}
$$


and we can find the expression for the inversion,

$$
A_{i}^{a}(x)=-\frac{1}{2} \epsilon^{a b c} u^{b k}(x) \nabla_{i} u_{k}^{c}(x)
$$

We will now compute a generic variation of this equation, so that we can later use it to compute the inversion for the gluino field. In order to carry out the calculation, we will need to know what is the generic variation of the connection (4.12). Using the fact that it is metric compatible, this can be computed to be,

$$
\delta \Gamma_{j k}^{n}=\frac{1}{2} g^{n m}\left(\nabla_{j} \delta g_{m k}+\nabla_{k} \delta g_{m j}-\nabla_{m} \delta g_{j k}\right)-\delta K_{j k}^{n}
$$

We can now carry out the variation of the dreibein postulate, and from there obtain the variation of equation (4.15). The result will be,

$$
\begin{gathered}
\delta A_{i}^{a}=\frac{\epsilon^{n m l}}{2 \sqrt{g}} u_{m}^{a}\left(\nabla_{i}\left(u_{l}^{b} \delta u_{n}^{b}\right)+\nabla_{l}\left(\delta g_{n i}\right)+\frac{i}{2}\left(\left(\bar{\psi}_{i} \gamma^{b} \psi_{l}\right) \delta u_{n}^{b}+\frac{1}{2}\left(\bar{\psi}_{n} \gamma^{b} \psi_{l}\right) \delta u_{i}^{b}\right)+\right. \\
\left.+\frac{i}{2}\left(\bar{\psi}_{n} \gamma_{l} \delta \psi_{i}+\bar{\psi}_{i} \gamma_{n} \delta \psi_{l}+\bar{\psi}_{n} \gamma_{i} \delta \psi_{l}\right)\right)
\end{gathered}
$$

where $\sqrt{g}=\operatorname{det} u$.

We will now use this equation to invert for the gluino field. As we know the geometrical variation (4.3) with $\xi \equiv \varepsilon$ generates the bosonic supersymmetry variation. So, we only need to use (2.3) and (4.3) in (4.17), and rearrange, so that we find the expression for the inversion,

$$
\lambda^{a}(x)=-\frac{\epsilon^{n m l}}{6 \sqrt{g(x)}} u_{m}^{a}(x) \gamma^{i}(x)\left(\gamma_{l}(x) \mathcal{D}_{i} \psi_{n}(x)+\gamma_{n}(x) \mathcal{D}_{l} \psi_{i}(x)+\gamma_{i}(x) \mathcal{D}_{l} \psi_{n}(x)\right)
$$

where we use the vector-spinor full covariant derivative, defined as,

$$
\mathcal{D}_{i} \psi_{k \alpha} \equiv \partial_{i} \psi_{k \alpha}+\frac{1}{2} \omega_{i}^{a b}\left(\sigma^{a b}\right)_{\alpha \beta} \psi_{k \beta}-\Gamma_{i k}^{s} \psi_{s \alpha}
$$

Observe that even though the spin-connection is defined via the gauge connection, it is a fully geometric quantity through the dreibein postulate. Later on we will also require an expression for the generic variation of this equation, so we will address such a problem now. The computation is rather long, and so is the result. We will obtain,

$$
\begin{gathered}
\delta \lambda^{a}=-\frac{\epsilon^{n m l}}{6 \sqrt{g}} u_{m}^{a}\left(u^{b i} \delta u_{l}^{c}+u_{l}^{c} \delta u^{b i}\right) \gamma^{b} \gamma^{c}\left(\mathcal{D}_{i} \psi_{n}-\mathcal{D}_{n} \psi_{i}\right)- \\
-\frac{\epsilon^{n m l}}{6 \sqrt{g}} u_{m}^{a} \gamma^{i}\left(\gamma_{l} \mathcal{D}_{i}\left(\delta \psi_{n}\right)+\gamma_{n} \mathcal{D}_{l}\left(\delta \psi_{i}\right)+\gamma_{i} \mathcal{D}_{l}\left(\delta \psi_{n}\right)-\right.
\end{gathered}
$$




$$
\begin{gathered}
-\frac{1}{2}\left(\gamma_{l} \sigma^{j k} \psi_{n} \nabla_{i}+\gamma_{n} \sigma^{j k} \psi_{i} \nabla_{l}+\gamma_{i} \sigma^{j k} \psi_{n} \nabla_{l}\right)\left(u_{j}^{b} \delta u_{k}^{b}\right)- \\
-\frac{1}{2}\left(\gamma_{l} \sigma^{j k} \psi_{n} \nabla_{j}\left(\delta g_{k i}\right)+\gamma_{n} \sigma^{j k} \psi_{i} \nabla_{j}\left(\delta g_{k l}\right)+\gamma_{i} \sigma^{j k} \psi_{n} \nabla_{j}\left(\delta g_{k l}\right)\right)+ \\
\left.+\frac{1}{2}\left(\gamma_{l} \sigma^{j k} \psi_{n} \delta K_{i j k}+\gamma_{n} \sigma^{j k} \psi_{i} \delta K_{l j k}+\gamma_{i} \sigma^{j k} \psi_{n} \delta K_{l j k}\right)+\left(\gamma_{l} \delta K_{i n}{ }^{s}+\gamma_{n} \delta K_{l i}{ }^{s}+\gamma_{i} \delta K_{l n}{ }^{s}\right) \psi_{s}\right)- \\
-\frac{1}{6 \sqrt{g}} u_{j}^{a} u_{k}^{c}\left(\epsilon^{n j k} \delta u^{c l}-\epsilon^{l j k} \delta u^{c n}\right) \gamma^{i}\left(\gamma_{l} \mathcal{D}_{i} \psi_{n}+\gamma_{n} \mathcal{D}_{l} \psi_{i}+\gamma_{i} \mathcal{D}_{l} \psi_{n}\right)
\end{gathered}
$$

where the generic variation of the contorsion tensor can be written as,

$$
\begin{gathered}
\delta K_{i n l}=\frac{i}{4}\left(\left(\bar{\psi}_{i} \gamma^{a} \psi_{l}\right) \delta u_{n}^{a}+\left(\bar{\psi}_{n} \gamma^{a} \psi_{l}\right) \delta u_{i}^{a}-\left(\bar{\psi}_{i} \gamma^{a} \psi_{n}\right) \delta u_{l}^{a}\right)+ \\
+\frac{i}{4}\left(\left(\bar{\psi}_{n} \gamma_{l}-\bar{\psi}_{l} \gamma_{n}\right) \delta \psi_{i}+\left(\bar{\psi}_{i} \gamma_{n}+\bar{\psi}_{n} \gamma_{i}\right) \delta \psi_{l}-\left(\bar{\psi}_{l} \gamma_{i}+\bar{\psi}_{i} \gamma_{l}\right) \delta \psi_{n}\right)
\end{gathered}
$$

The variations (4.17) and (4.20) allow us now to express a variation of the wavefunctional in terms of the variations of the geometric variables. This will be helpful in section 5 .

The inversion completed proves the non existence of Wu-Yang ambiguities in the new geometrical variables. Therefore, we have managed to define new gauge invariant variables for supersymmetric Yang-Mills. Moreover, it can be shown that gauge invariant physical wave-functionals of the theory depend only on these geometric variables (see section 5 ), $\Psi \equiv \Psi\left[g_{i j}, \psi_{k}\right]$, so that we have in these variables an explicit parameterization of the physical Hilbert space (moduli space) of the gauge theory. A final remark on diffeomorphisms is now in order. As said before, only the variables of the theory are diffeomorphism covariant. The Hamiltonian fails to be diffeomorphism

covariant. Given that the variables of the theory are now $\left\{g_{i j}, \psi_{k}\right\}$, this has an interesting consequence: a configuration diffeomorphic to the previous one yields a different configuration to the gauge theory. Therefore, we can extend solutions to the gauge theory by action of the group of diffeomorphisms, by simply moving along the orbit of the geometrical configuration.

\section{Gauge Tensors as Geometric Tensors}

We now wish to write the tensors and composite operators of our theory in terms of the new geometric variables, i.e., as geometric tensors and geometric composite operators. We will first address the electric and magnetic tensors. The Hamiltonian, Gauss' law generator, and the supersymmetry generator composite operators then easily follow from these two tensors and the previous equations for the inversions of the gluon and gluino fields.

Let us start with the gauge Ricci identity,

$$
\epsilon^{a b c} F_{i j}^{b}=\left[D_{i}, D_{j}\right]^{a c}
$$


and apply it to the dreibein field. We will obtain,

$$
\epsilon^{a b c} F_{i j}^{b} u_{k}^{c}=R_{k i j}^{l} u_{l}^{a}
$$

where $R_{k i j}^{l}$ is the Riemann tensor of the connection $\Gamma$,

$$
R_{k i j}^{l}=\partial_{i} \Gamma_{j k}^{l}-\partial_{j} \Gamma_{i k}^{l}+\Gamma_{j k}^{m} \Gamma_{i m}^{l}-\Gamma_{i k}^{m} \Gamma_{j m}^{l}
$$

Now from (5.2) we can express the field strength in terms of the Riemann curvature, and so from (3.3) we can express the magnetic field vector geometrically, as,

$$
B^{a m}=-\frac{1}{4 \sqrt{g}} \epsilon^{m i j} \epsilon^{n l k} u_{n}^{a} R_{l k i j}
$$

So, the gauge invariant tensor which gives the Yang-Mills magnetic energy density is,

$$
B^{a i} B^{a j}=\frac{1}{16} \epsilon^{i m n} \epsilon^{j k l} R_{m n}^{u v}\left(R_{u v k l}-R_{v u k l}\right)
$$

As we can see, this expression gives the gauge invariant tensor in a manifestly gauge invariant form, in terms of the "metric" $g_{i j}$, and the "gravitino" $\psi_{k}$ (which is present via the torsion contribution to the Riemann tensor).

The electric field vector is the momentum canonically conjugated to the canonical variable, the gauge connection. In canonical quantization it is represented by a functional derivative (3.9). We will define a gauge invariant tensor operator $e^{i j}$ by,

$$
\frac{\delta}{\delta A_{i}^{a}(x)}=i E^{a i}(x) \equiv \sqrt{g(x)} u_{j}^{a}(x) e^{i j}(x)
$$

Clearly, $e^{i j}(x)$ is an ordinary $\left(\begin{array}{l}2 \\ 0\end{array}\right)$ tensor under $G L(3)$. From this expression, the electric gauge invariant Yang-Mills tensor, i.e., the manifestly gauge invariant tensor which gives the Yang-Mills electric energy density, now follows as,

$$
E^{a i} E^{a j}=-g e_{k}^{i} e^{j k}
$$

In order to finally obtain the Hamiltonian in a manifestly gauge invariant form in terms of the geometrical variables, we still need the expression for the fermionic energy density, as is clear from (3.1). The expression for this gauge invariant tensor can be obtained by simply inserting (4.18-19) in the required expression. The result we will obtain is,

$$
\begin{gathered}
\bar{\lambda}^{a} \gamma^{i} D_{i} \lambda^{a}=\frac{\epsilon^{n j k}}{36 \sqrt{g}} g_{j m}\left(\left(\overline{\mathcal{D}}_{k} \bar{\psi}_{n}\right) \gamma_{l}+\left(\overline{\mathcal{D}}_{l} \bar{\psi}_{n}\right) \gamma_{k}+\right. \\
\left.+\left(\overline{\mathcal{D}}_{k} \bar{\psi}_{l}\right) \gamma_{n}\right) \gamma^{l} \gamma^{i} \nabla_{i}\left(\frac{\epsilon^{u m v}}{\sqrt{g}} \gamma^{s}\left(\gamma_{v}\left(\mathcal{D}_{s} \psi_{u}\right)+\gamma_{u}\left(\mathcal{D}_{v} \psi_{s}\right)+\gamma_{s}\left(\mathcal{D}_{v} \psi_{u}\right)\right)\right)
\end{gathered}
$$


where we have defined $\overline{\mathcal{D}}_{i} \bar{\psi}_{j} \equiv\left(\mathcal{D}_{i} \psi_{j}\right)^{\dagger} \gamma^{0}$; and where in the contraction $\gamma^{i} \nabla_{i}$ the gamma matrices are to be considered as numerical, not as space dependent. The sum of (5.5), (5.7) and (5.8) according to (3.1) finally yields the manifestly gauge invariant Hamiltonian.

As was done for the gluon functional derivative, we will now similarly define a gauge invariant vector-spinor density operator $\chi_{i}$ to deal with the gluino functional derivative,

$$
\frac{\delta}{\delta \lambda^{a}(x)} \equiv \sqrt{g(x)} u^{a i}(x) \chi_{i}(x)
$$

$\chi_{i}(x)$ is a $\left(\begin{array}{l}0 \\ 1\end{array}\right)$ vector-spinor density (weight $\frac{1}{2}$ ) under $G L(3)$. With these definitions at hand, we can now express the functional dependence of the wave-functional $\Psi\left[A_{i}^{a}, \lambda^{b}\right]$ in terms of the new variables. Under a variation, we will have,

$$
\begin{gathered}
\delta \Psi=\int d^{3} x\left\{\frac{\delta \Psi}{\delta A_{i}^{a}(x)} \delta A_{i}^{a}(x)+\frac{\delta \Psi}{\delta \lambda^{a}(x)} \delta \lambda^{a}(x)\right\}= \\
=\int d^{3} x\left\{\sqrt{g(x)} u_{j}^{a}(x) \delta A_{i}^{a}(x)\left[e^{i j}(x) \Psi\right]+\sqrt{g(x)} u^{a i}(x)\left[\chi_{i}(x) \Psi\right] \delta \lambda^{a}(x)\right\}
\end{gathered}
$$

where we should use the expression for the variations of the gauge fields in (4.17) and (4.20-21). Expanding this expression through rather lengthy calculations, it can then be seen that the term in $\delta u_{i}^{a}$ is proportional to the Gauss' law operator (3.4), when expressed in geometrical terms, and acting on the wave-functional. Observe that this is $\mathcal{G}^{a} \Psi[g, \psi]=0$, which in the new variables can be written as,

$$
\begin{gathered}
\left(\nabla_{i} e^{i j}+\frac{i}{2} \bar{\psi}_{i} \gamma^{k} \psi_{k} e^{i j}+\frac{1}{72 g^{2}}\left(g^{j n} \epsilon^{i l k}-g^{j l} \epsilon^{i n k}\right)\left(\left(\overline{\mathcal{D}}_{s} \bar{\psi}_{n}\right) \gamma_{l}+\left(\overline{\mathcal{D}}_{l} \bar{\psi}_{s}\right) \gamma_{n}+\right.\right. \\
\left.\left.+\left(\overline{\mathcal{D}}_{l} \bar{\psi}_{n}\right) \gamma_{s}\right) \gamma^{s} \gamma^{0} \gamma^{r}\left(\gamma_{k}\left(\mathcal{D}_{r} \psi_{i}\right)+\gamma_{i}\left(\mathcal{D}_{k} \psi_{r}\right)+\gamma_{r}\left(\mathcal{D}_{k} \psi_{i}\right)\right)\right) \Psi[g, \psi]=0
\end{gathered}
$$

So, wave-functionals whose dependence is solely on the new gauge invariant variables are gauge invariant, and gauge invariant wave-functionals depend solely on the new gauge invariant variables. It is in these physical gauge invariant wave-functionals that we are mainly interested, and for these the previous expression for $\delta \Psi$ reduces to,

$$
\begin{gathered}
\delta \Psi\left[g_{i j}, \psi_{k}\right]=\int d^{3} x\left\{\frac{1}{2} \epsilon^{n m l} \nabla_{l}\left(\delta g_{n i}\right)\left[e_{m}^{i} \Psi\right]+\right. \\
+\frac{1}{12} \epsilon^{n m l}\left[\chi_{m} \Psi\right] \gamma^{i}\left(\gamma_{l} \sigma^{j k} \psi_{n} \nabla_{j}\left(\delta g_{k i}\right)+\gamma_{n} \sigma^{j k} \psi_{i} \nabla_{j}\left(\delta g_{k l}\right)+\gamma_{i} \sigma^{j k} \psi_{n} \nabla_{j}\left(\delta g_{k l}\right)\right)+ \\
+\frac{i}{4} \epsilon^{n m l}\left(\bar{\psi}_{n} \gamma_{l} \delta \psi_{i}+\bar{\psi}_{i} \gamma_{n} \delta \psi_{l}+\bar{\psi}_{n} \gamma_{i} \delta \psi_{l}\right)\left[e_{m}^{i} \Psi\right]- \\
-\frac{1}{6} \epsilon^{n m l}\left[\chi_{m} \Psi\right] \gamma^{i}\left(\gamma_{l} \mathcal{D}_{i}\left(\delta \psi_{n}\right)+\gamma_{n} \mathcal{D}_{l}\left(\delta \psi_{i}\right)+\gamma_{i} \mathcal{D}_{l}\left(\delta \psi_{n}\right)\right)- \\
-\frac{i}{24} \epsilon^{n m l}\left[\chi_{m} \Psi\right] \gamma^{i}\left(\gamma_{l} \sigma^{j k} \psi_{n}\left(\bar{\psi}_{j} \gamma_{k} \delta \psi_{i}+\bar{\psi}_{i} \gamma_{j} \delta \psi_{k}+\bar{\psi}_{j} \gamma_{i} \delta \psi_{k}\right)+\right.
\end{gathered}
$$




$$
\begin{gathered}
\left.+\gamma_{n} \sigma^{j k} \psi_{i}\left(\bar{\psi}_{j} \gamma_{k} \delta \psi_{l}+\bar{\psi}_{l} \gamma_{j} \delta \psi_{k}+\bar{\psi}_{j} \gamma_{l} \delta \psi_{k}\right)+\gamma_{i} \sigma^{j k} \psi_{n}\left(\bar{\psi}_{j} \gamma_{k} \delta \psi_{l}+\bar{\psi}_{l} \gamma_{j} \delta \psi_{k}+\bar{\psi}_{j} \gamma_{l} \delta \psi_{k}\right)\right)- \\
\left.-\frac{i}{12} \epsilon^{n m l}\left[\chi_{m} \Psi\right] \gamma^{i}\left(\gamma_{l} \psi_{s}\left(\bar{\psi}_{n} \gamma^{s} \delta \psi_{i}\right)+\gamma_{n} \psi_{s}\left(\bar{\psi}_{i} \gamma^{s} \delta \psi_{l}\right)+\gamma_{i} \psi_{s}\left(\bar{\psi}_{n} \gamma^{s} \delta \psi_{l}\right)\right)\right\}
\end{gathered}
$$

From here we can now extract expressions for the electric and spinor fields, $e^{i j} \Psi$ and $\chi_{i} \Psi$, in terms of functional derivatives of gauge invariant wave-functionals, with respect to the gauge invariant variables. Observe that for that, we have to solve a linear system of differential equations, therefore involving the inversion of differential operators. We can then conclude that in general, both operators $e^{i j} \Psi$ and $\chi_{i} \Psi$, will depend non-locally on the functional derivatives $\delta \Psi / \delta g_{i j}$ and $\delta \Psi / \delta \psi_{k}$. Like in the non-supersymmetric case [8], the Hamiltonian will thus be a non-local composite operator.

Before proceeding with the study of these non-local operators, there is one more composite operator that we still would like to express in a manifestly gauge invariant way, i.e., that we would like to geometrize. Such an operator is the supersymmetry generator, (3.5-6). In particular, we will look at its structure as depicted in equations (3.5),(3.25-26), and geometrize the tensor $\amalg^{i}(x)$. For that, we simply have to make use of the previous formulae into equation (3.26), and obtain,

$$
\begin{gathered}
\amalg^{i}=\left(\begin{array}{cc}
0 & -\frac{e}{6} \epsilon^{n m l} e_{m}^{i}+\frac{1}{24 e g} \epsilon^{i j k}\left(R^{n l}{ }_{j k}-R^{l n}{ }_{j k}\right) \\
\frac{e}{6} \epsilon^{n m l} e_{m}^{i}+\frac{1}{24 e g} \epsilon^{i j k}\left(R^{n l}{ }_{j k}-R^{l n}{ }_{j k}\right) & 0
\end{array}\right. \\
\cdot \gamma^{r}\left(\gamma_{l} \mathcal{D}_{r} \psi_{n}+\gamma_{n} \mathcal{D}_{l} \psi_{r}+\gamma_{r} \mathcal{D}_{l} \psi_{n}\right)
\end{gathered}
$$

from where the supersymmetry generator then follows, according to (3.25) and (3.5).

Some words are now in order, concerning the supersymmetry algebra and its quantum field theoretic representation on the geometrized fields. One of the elements that is present in $\amalg^{i}$ is the non-local operator $e^{i j}$, thus turning the supersymmetry generator into a non-local composite operator, when expressed in the geometrical variables. As we will see in the following, information about the Green's functions present in this operator can be obtained, albeit in a formal way. By this, we mean that an explicit construction of these Green's functions can only be obtained given a particular geometrical configuration (see [12] for this same situation in the nonsupersymmetric case). Precisely due to this reason, it follows that the geometric quantum field theoretic representation of the supersymmetry algebra can only be explicitly presented once we have chosen a particular geometrical configuration, and so have explicit knowledge of the Green's functions present in the non-local operators.

Moreover, the geometric supersymmetry generator includes the Riemann tensor which is non-linear in the metric and "gravitino" fields, and their derivatives; we would therefore also prefer to have a geometrical configuration with a high degree of symmetry (a maximal number of Killing vectors), in order to simplify it. An example involving spherical geometries, generalizing the one in [12] to this supersymmetric case, shows how this situation is handled explicitly 22]. 
In the pure Yang-Mills case [8], the calculation of the electric field tensor involved the inversion of a differential operator that could generically have zero modes. Subtleties associated to the inversion of such an operator were later handled with the insertion of a deformation into the dreibein defining equation 12]. We will now see that in this supersymmetric case those problems can be better handled, by computing the bosonic Green's function for the electric field tensor $e^{i j}$. We will see that we will not need to deform our equations in order to obtain a well-defined result. We start by inverting the defining equation for the electric tensor (5.6), to obtain,

$$
e^{i j}=\frac{1}{\sqrt{g}} u^{a j} \frac{\delta}{\delta A_{i}^{a}}
$$

Recall that through the dreibein defining equation (4.6), the gauge connection depends on both the dreibein and the "gravitino" field. Therefore, we can further expand the geometric tensor $e^{i j}$ as,

$$
e^{i j}(x)=\frac{1}{\sqrt{g(x)}} u^{a j}(x) \int d^{3} y\left(\frac{\delta u_{k}^{b}(y)}{\delta A_{i}^{a}(x)} \frac{\delta}{\delta u_{k}^{b}(y)}+\frac{\delta \psi_{k}(y)}{\delta A_{i}^{a}(x)} \frac{\delta}{\delta \psi_{k}(y)}\right)
$$

Variations of the dreibein can be further separated into variations of the six gauge invariant degrees of freedom $g_{i j}$ and of the three gauge degrees of freedom. As we are considering operators that act on gauge invariant wave-functionals only, we will simply obtain,

$$
e^{i j}(x)=\int d^{3} y \frac{1}{\sqrt{g(x)}}\left\{\left(u^{a j}(x) \frac{\delta u_{k}^{b}(y)}{\delta A_{i}^{a}(x)} u_{m}^{b}(y)\right) 2 \frac{\delta}{\delta g_{k m}(y)}+u^{a j}(x) \frac{\delta \psi_{k}(y)}{\delta A_{i}^{a}(x)} \frac{\delta}{\delta \psi_{k}(y)}\right\}
$$

We now want to study the bosonic Jacobian matrix $\delta u / \delta A$, and see that it has a better behavior in here, than in the non-supersymmetric Yang-Mills case. For that, we need to start by geometrizing such a matrix. Let us re-write (4.6) as,

$$
\epsilon^{i j k}\left(\delta^{a c} \delta_{k}^{n} \partial_{j}+\delta_{k}^{n} \epsilon^{a b c} A_{j}^{b}-\frac{i}{4} \delta^{a c}\left(\bar{\psi}_{j} \gamma^{n} \psi_{k}\right)\right) u_{n}^{c}=0
$$

Variation of this equation is (4.8-9),

$$
\epsilon^{i j k} D_{j} \delta u_{k}^{a}=-\epsilon^{i j k} \epsilon^{a b c} u_{k}^{c} \delta A_{j}^{b}+\frac{i}{2} \epsilon^{i j k} \bar{\psi}_{j} \gamma^{a} \delta \psi_{k}
$$

and to obtain $\delta u_{i}^{a}$ in terms of $\delta A_{i}^{a}$, the operator acting on $\delta u_{i}^{a}$ must be inverted. In order to do so, let us consider the associated eigenvalue problem,

$$
\epsilon^{i j k}\left(\delta^{a c} \delta_{k}^{n} \partial_{j}+\delta_{k}^{n} \epsilon^{a b c} A_{j}^{b}-\frac{i}{4} \delta^{a c}\left(\bar{\psi}_{j} \gamma^{n} \psi_{k}\right)\right) w_{A_{n}^{c}}^{c}=\sqrt{g} \Lambda_{A} w_{A}^{i a}
$$

By definition, one solution to this equation with $\Lambda_{A}=0$ is $u_{i}^{a}$ itself. In our notation, $A$ labels all the eigenfunctions, except the particular one given by $u_{i}^{a}$. Moreover, 
it will be assumed that $\left\{u_{i}^{a}, w_{A_{i}}^{a}\right\}$ forms a complete orthonormal spectrum of real eigenfunctions for the considered operator. By orthonormality, we mean,

$$
\begin{gathered}
\int \sqrt{g} d^{3} x g^{i j}\left(u_{i}^{a} w_{A_{j}}^{a}\right)=0 \\
\int \sqrt{g} d^{3} x g^{i j}\left(w_{A_{i}}^{a} w_{B j}^{a}\right)=3 V \delta_{A B} \\
\int \sqrt{g} d^{3} x g^{i j}\left(u_{i}^{a} u_{j}^{a}\right)=3 V
\end{gathered}
$$

where $V$ is the volume of the space described by $g_{i j}$ (i.e., $V$ is a "dynamical" volume), and $\delta_{A B}$ is a Kronecker or Dirac delta, depending on wether the spectrum is discrete or continuous. Now, expand a generic variation of the dreibein in this complete set,

$$
\delta u_{i}^{a}=\eta u_{i}^{a}+\sum_{A} \eta_{A} w_{A i}^{a}
$$

and substitute this in (5.18). If we dot on the left (meaning inner product with the required measure (5.20)) with the same complete set $\left\{u_{i}^{a}, w_{A_{i}^{a}}^{a}\right\}$, we will obtain a non-homogeneous linear system of equations for the expansion parameters, $\eta$ and $\eta_{A}$. Solving that system, and inserting the result in (5.21) yields an expansion of the variation $\delta u_{i}^{a}$ in terms of the variations $\delta A_{i}^{a}$ and $\delta \psi_{k}$. It is then easy to compute the Jacobian matrix $\delta u / \delta A$. However, such a result will not be naturally geometric, as it involves the eigenfunctions $w_{A_{i}}^{a}$, which are gauge vectors. To solve this problem, we now introduce the geometric modes $z_{A i}{ }^{j}$, associated to the gauge modes $w_{A i}{ }^{a}$, and defined via,

$$
w_{A i}^{a} \equiv z_{A i}^{j} u_{j}^{a}
$$

It can then be shown that these geometric modes obey,

$$
\epsilon^{i j k} \nabla_{j} z_{A k}^{m}=\sqrt{g} \Lambda_{A} z_{A}^{i m}
$$

So, the $z_{A}{ }^{i j}$ are the eigenmodes of the geometric curl operator, with the same eigenvalues as the gauge modes $w_{A i}^{a}, \Lambda_{A}$. Full geometrization of the Green's function,

$$
u^{a j}(x) \frac{\delta u_{k}^{b}(y)}{\delta A_{i}^{a}(x)} u_{m}^{b}(y)
$$

is now at hand. The result is,

$$
\begin{gathered}
u^{a j}(x) \frac{\delta u_{k}^{b}(y)}{\delta A_{i}^{a}(x)} u_{m}^{b}(y)=\sqrt{g(x)} g^{i j}(x)\left\{\mathcal{H}_{k m s}{ }^{s}(y, x)-\right. \\
-\frac{3}{\int d^{3} x \epsilon^{i j k}\left(\bar{\psi}_{j} \gamma_{i} \psi_{k}\right)} \int d^{3} u \epsilon^{i j k}\left(\bar{\psi}_{j} \gamma_{n} \psi_{k}\right)(u) \mathcal{H}_{k m i}{ }^{n}(y, u)+
\end{gathered}
$$




$$
\begin{gathered}
+g_{k m}(y) \frac{8 i}{\int d^{3} x \epsilon^{i j k}\left(\bar{\psi}_{j} \gamma_{i} \psi_{k}\right)}\left\{3+\frac{i}{8} \int d^{3} u \epsilon^{i j k}\left(\bar{\psi}_{j} \gamma^{n} \psi_{k}\right)(u) \mathcal{H}_{n i s}{ }^{s}(u, x)-\right. \\
\left.\left.-\frac{3 i}{8 \int d^{3} x \epsilon^{i j k}\left(\bar{\psi}_{j} \gamma_{i} \psi_{k}\right)} \iint d^{3} u d^{3} v \epsilon^{i j k}\left(\bar{\psi}_{j} \gamma^{n} \psi_{k}\right)(u) \epsilon^{m r s}\left(\bar{\psi}_{r} \gamma_{l} \psi_{s}\right)(v) \mathcal{H}_{n i m}{ }^{l}(u, v)\right\}\right\}
\end{gathered}
$$

where we have defined the Green's functions,

$$
\mathcal{H}_{i j m n}(x, y) \equiv \sum_{A B} z_{A i j}(x) I_{A B}^{-1} z_{B m n}(y)
$$

and the matrix,

$$
\begin{gathered}
I_{A B} \equiv-\frac{3}{2} V \Lambda_{A} \delta_{A B}-\frac{i}{8} \int d^{3} x \epsilon^{i j k}\left(\bar{\psi}_{j} \gamma^{n} \psi_{k}\right) z_{A i m} z_{B n}{ }^{m}+ \\
+\frac{i}{8} \frac{1}{\int d^{3} x \epsilon^{i j k}\left(\bar{\psi}_{j} \gamma_{i} \psi_{k}\right)}\left(\int d^{3} x \epsilon^{i j k}\left(\bar{\psi}_{j} \gamma_{l} \psi_{k}\right) z_{A i}{ }^{l}\right)\left(\int d^{3} x \epsilon^{i j k}\left(\bar{\psi}_{j} \gamma^{n} \psi_{k}\right) z_{B n i}\right)
\end{gathered}
$$

We see that we have obtained a well-defined result, unlike it would have happen in the non-supersymmetric case [8, where there were divergences in the electric energy that were independent of the geometry. Clearly, the Green's functions (5.26) may still have geometry dependent divergences associated with the degree of symmetry of a given geometrical configuration (as determined by its Killing vectors). Also, this may seem a somewhat formal result, but observe that now we have a constructive definition of the Green's function (5.24): given a geometrical configuration on the domain manifold, we start by solving the eigenvalue equation in order to obtain the geometric eigenmodes. Once we have such eigenmodes, we first construct the matrix $I_{A B}$, then invert it (most likely through a symbolic manipulation program), and finally compute the Green's functions $\mathcal{H}_{i j m n}(x, y)$.

We have now geometrized all the tensors appearing in the Hamiltonian formulation of $N=1$ supersymmetric Yang-Mills theory. All composite operators should now follow in a straightforward fashion.

\section{Conclusions}

We have defined new local gauge invariant variables for supersymmetric gauge theory. These variables have moreover a geometrical interpretation, as they are a three dimensional "metric" and a "gravitino". The geometry associated to the theory is then just like the geometry of supergravity.

We have also shown that these new variables are free of Wu-Yang ambiguities; so they seem to be quite appropriate for the study of nonperturbative phenomena in supersymmetric gauge theories, as they explicitly parameterize the physical Hilbert space of the theory. We have also seen that these variables have a better behavior here 
than in the non-supersymmetric case. Namely, there are no geometry independent divergences in the bosonic half of the electric energy tensor operator.

The treatment presented here was rather formal, and the issue of renormalization was not addressed. Further work on this formalism should focus on this problem. We could think of using the known beta functions of supersymmetric Yang-Mills theory and perform the renormalization of the (geometric) composite operators that we have presented. These renormalized operators could then be used to extract information on the ground state wave-functional of the gauge theory. In this supersymmetric case, the functional differential equation for the ground state is $Q \Psi[g, \psi]=0$, which is first-order in the non-local functional derivatives. There would seem to be hope that we could then extract some information about the solution to the theory.

It would also be interesting to study special solutions to supersymmetric YangMills theory in this framework. Namely, we could try to extend to this supersymmetric case the example of spherical geometries that was introduced in [12]. In particular, in order to define a vector-spinor on a three manifold, the manifold must be parallelisable, and its second Stiefel-Whitney cohomology class must be trivial. Such is the case for $S^{3}$, so that the example in [12] could indeed be generalizable to this framework [22]. There is also the possibility of extending this formalism to higher $N$ supersymmetric gauge theory. This could be interesting, specially if some connection to the work in [19] could then be established.

All these lines of work are quite interesting to follow as there is good knowledge about some properties of supersymmetric Yang-Mills theories (see 23] for a modern review, and references therein). In the example of spherical geometries, a bridge between our formalism and the well known instanton solutions of gauge theory can be established; while in the case of extended $N=2$ supersymmetric Yang-Mills theory, we can observe the interesting fact that BPS states obey - in the geometrical formulation - a three dimensional "Einstein field equation" where the "stress tensor" is the one associated to a gauge vector field [22].

One well known - and exact - result from supersymmetric Yang-Mills theory is that the gluino condensate $\langle\lambda \lambda\rangle$ develops a non-zero vacuum expectation value, thus spontaneously breaking the discrete symmetry of the model, $Z_{2 N}$, down to $Z_{2}$. There will then be $N$ degenerate vacua, all with vanishing energy density [23. In order to reproduce such a result in this geometrical formalism we need to set up the calculation of the gluino condensate, and for that we need a trial wave-functional in order to obtain the $\langle\lambda \lambda\rangle$ vacuum expectation value in this Hamiltonian formulation. So again, we will have to restrict ourselves to a particular geometrical configuration in order to perform the calculation. An interesting fact here is that the result for the gluino condensate 23] comes from a one-instanton calculation, and so it would be very interesting to know if the spherical geometry configuration can reproduce such a result. We hope to report on these questions in the near future 22]. 


\section{Acknowledgements}

I would like to thank Kenneth Johnson for many discussions and advice, and Peter Haagensen for comments and reading of the manuscript. The author is partially supported by the Praxis XXI grant BD-3372/94 (Portugal).

\section{References}

[1] M.S. Narasimhan \& T.R. Ramadas, Geometry of SU(2) Gauge Fields, Comm. Math. Phys. 67 (1979) 121-136.

[2] O. Babelon \& C.M. Viallet, The Riemannian Geometry of the Configuration Space of Gauge Theories, Comm. Math. Phys. 81 (1981) 515-525.

[3] M.B. Halpern, Gauge Invariant Formulation of the Seldual Sector, Phys. Rev. D16 (1977) 3515-3519.

[4] J. Goldstone \& R. Jackiw, Unconstrained Temporal Gauge for Yang-Mills Theory, Phys. Lett. 74B (1978) 81-84.

[5] D.Z. Freedman, P.E. Haagensen, K. Johnson \& J.I. Latorre, The Hidden Spatial Geometry of Nonabelian Gauge Theories, hep-th/9309045.

[6] M. Bauer, D.Z. Freedman \& P.E. Haagensen, Spatial Geometry of the Electric Field Representation of Nonabelian Gauge Theories, Nucl. Phys. B428 (1994) 147-168, hep-th/9405028.

[7] F.A. Lunev, Four Dimensional Yang-Mills Theory in Local Gauge Invariant Variables, Mod. Phys. Lett. A9 (1994) 2281-2292, hep-th/9407175.

[8] P.E. Haagensen \& K. Johnson, Yang-Mills Fields and Riemannian Geometry, Nucl. Phys. B439 (1995) 597-616, hep-th/9408164.

[9] F.A. Lunev, Reformulation of QCD in the Language of General Relativity, J. Math. Phys. 37 (1996) 5351-5367, hep-th/9503133.

[10] P.E. Haagensen, New Gauge Invariant Variables for Yang-Mills Theory, Meeting on High Energy Physics, Rochester (1995), hep-th/9505188.

[11] O. Ganor \& J. Sonnenschein, The "Dual"Variables of Yang-Mills Theory and Local Gauge Invariant Variables, Int. J. Mod. Phys. A11 (1996) 5701-5728, hep-th/9507036.

[12] P.E. Haagensen, K. Johnson \& C.S. Lam, Gauge Invariant Geometric Variables for Yang-Mills Theory, Nucl. Phys. B477 (1996) 273-292, hep-th/9511226. 
[13] P.E. Haagensen \& K. Johnson, On the Wavefunctional for Two Heavy Color Sources in Yang-Mills Theory, hep-th/9702204.

[14] T.T. Wu \& C.N. Yang, Concept of Nonintegrable Phase Factors and Global Formulation of Gauge Fields, Phys. Rev. D12 (1975) 3845-3857.

[15] R. Jackiw, Analysis on Infinite Dimensional Manifolds - Schroedinger Representation for Quantized Fields, Brazil Summer School (1989).

[16] D. Bailin \& A. Love, Supersymmetric Gauge Field Theory and String Theory, Institute of Physics Publishing (1994).

[17] J. Wess \& J. Bagger, Supersymmetry and Supergravity, Princeton Series in Physics (1992).

[18] P. West, Introduction to Supersymmetry and Supergravity, World Scientific (1990).

[19] N. Seiberg \& E. Witten, Electric-Magnetic Duality, Monopole Condensation, and Confinement in $N=2$ Supersymmetric Yang-Mills Theory, Nucl. Phys. B426 (1994) 19-52, hep-th/9407087.

[20] R. DiStefano, M. Kreuzer \& A. Rebhan, On the Canonical Formulation of Supersymmetric Yang-Mills Theories, Mod. Phys. Lett. A2 (1987) 487-497.

[21] R. DiStefano, Disappearance of the Auxiliary Fields in a Canonical Formulation of Supersymmetry, Phys. Lett. B192 (1987) 130-134.

[22] R. Schiappa, Work in progress.

[23] M. Shifman, Non-Perturbative Dynamics in Supersymmetric Gauge Theories, hep-th/9704114. 